\title{
The spun cusp complexified: complex ray focusing in chiral conical diffraction
}

\author{
M R Jeffrey \\ H H Wills Physics Laboratory, University of Bristol, Tyndall Avenue, Bristol BS8 1TL, UK \\ E-mail: mike.jeffrey@bristol.ac.uk
}

Received 23 March 2007, accepted for publication 3 May 2007

Published 19 June 2007

Online at stacks.iop.org/JOptA/9/634

\begin{abstract}
The intensity of light refracted along the optic axis of a slab of dichroic chiral birefringent crystal is studied for a Gaussian incident beam. The geometrical optics is derived in terms of complex rays. The dominant intensity features are exponential changes in intensity where pairs of rays exchange dominance, attributable to the interference of nonorthogonally polarized complex rays. These exponential gradients swamp the sites of ray focusing, which occur along two lines in space associated with the branch points of the ray equation, and around a closed loop called a complex whisker. The complex whisker intersects the branch focal lines at two 'complexified cusp' points, sites of higher-order focusing, which can be understood in terms of the complexification of one of the two control parameters defining the spun cusp catastrophe. The diffraction integrals are derived, and their asymptotics complementing the geometrical optics is explored.
\end{abstract}

Keywords: polarization, complex rays, chirality, dichroism, non-Hermitian

\section{Introduction}

A recent series of papers [1-5] have shown that conical diffraction-conical refraction of light by a biaxial crystal and the associated wave effects-arises in a domain where both Hamilton's geometric ray theory [6] and the full wave theory of Belskii and Khapalyuk [7] are essential to a proper understanding. Natural extensions to crystals with chirality (optical activity or the Faraday effect) [3] and dichroism [4] have required more sophisticated asymptotics and yielded a few surprises. Here we make the natural extension to include both effects combined.

In the presence of chirality the conical point of the wave surface [8], or degeneracy of the evolution operator matrix [1], responsible for conical refraction, is removed and replaced by a ring of inflection points in the wave surface $[9,10]$. This produces focusing along a circularly symmetric caustic surface, in the form of a spun cusp threaded by an axial focal line. Although the quartic order of the ray equation prevents a simple explicit expression of the ray intensity, geometrical optics still provides a detailed understanding of these dominant features [3], recognizable as singularities in the geometric intensity due to the coalescence of rays. These rays are the saddlepoints of the integrand in the full diffraction theory, and contributing saddlepoints are chosen if they lie along the unique path of integration for which the integral converges, when deformed to lie along contours of phase. In this way the caustic surface is found to enclose a region of space where four saddlepoints contribute as rays, two of which coalesce at the surface. Only three saddlepoints contribute in the region outside the caustic, two of which are rays and the third, decaying exponentially away from the surface, constitutes an evanescent wave.

The introduction of dichroism $[11,12]$ requires the notion of complex rays-solutions of the ray equation in complex coordinates as a function of a complex wavevector. The locus of such a complex ray has no corresponding path in real space, instead deriving its powerful meaning from its status as a saddlepoint through the method of stationary phase. For Gaussian beams this leads to a surprising duality between the effects of dichroism (nonhermiticity of the dielectric matrix and corresponding plane wave evolution operator) and beam misalignment (transition between conical and double refraction), which can be described by a single parameter [4]. Aside from an exponential ramp in the intensity present only with dichroism, the dominant features are geometrical interference, giving a dark cylindrical surface where two sets of rays exchange dominance, laced with a series of darker lines, characteristic of interference involving complex rays. 
Our aim here is to consider the effect of adding dichroism (equivalently beam misalignment) to chiral conical diffraction for a Gaussian incident beam. In section 2 we develop the geometrical optics, which involves complexifying the ray and wave coordinates of chiral conical diffraction. In section 3 we derive the full wave theory, and in section 4 we examine more closely its asymptotics, primarily the inclusion of phase into geometrical optics, and the complexification of the spun cusp - the rotationally symmetric extension of the Pearcey integral, whose asymptotics for complex variables were studied in [13].

Paraxially, that is within small angles of the optic axis, the birefringence of a crystal is specified by a single parameter, the half-angle of the conical refraction cone,

$$
A=\frac{1}{n_{2}} \sqrt{\left(n_{1}-n_{2}\right)\left(n_{2}-n_{3}\right)}
$$

which is the geometric mean of the crystal's principal refractive indices, $n_{1}<n_{2}<n_{3}$. A crystal slab of thickness $l$ (along the optic axis) and a Gaussian incident beam of waist width $w$ are completely specified by the radius of the refracted cone at the crystal's exit face measured in units of the beam width,

$$
\rho_{0} \equiv \frac{A l}{w}
$$

The effect of birefringence on a plane wavevector D, propagating paraxially close to an optic axis of the crystal, is the multiplication by a unitary matrix $\exp \left(\mathrm{iM}_{\mathrm{b}}\right)$, whose well known $[6,14,12]$ linear eigenpolarizations in the crystal rotate by a half-turn in a $2 \pi$ circuit of the optic axis. This determines the real symmetric matrix $M_{b}$ to be given, up to a trace, by

$$
\mathrm{M}_{\mathrm{b}}=-\rho_{0} w\left\{k_{x}, k_{y}\right\} \cdot\left\{\sigma_{3}, \sigma_{1}\right\},
$$

where $\left\{k_{x}, k_{y}\right\}$ is the (small) part of the wavevector transverse to the optic axis and the $\sigma_{\mathrm{i}}$ denote Pauli matrices.

Chirality and dichroism can also be specified by their effect on the polarization of a wave which has traversed the crystal along its optical axis. Consider an incident wave with circular polarization

$$
\mathbf{d}_{ \pm}^{\text {circ }}=\frac{1}{\sqrt{2}}\left(\begin{array}{c}
1 \\
\pm \mathrm{i}
\end{array}\right)
$$

Chirality changes the phase of a circularly polarized wave by a total amount $\gamma$, which we write in terms of a Hermitian matrix $\mathrm{M}_{\mathrm{ch}}$ as the eigenequation

$$
\mathbf{D}=\exp \left(\mathrm{iM}_{\mathrm{ch}}\right) \mathbf{d}_{ \pm}^{\mathrm{circ}}=\exp (\mp \mathrm{i} \gamma) \mathbf{d}_{ \pm}^{\mathrm{circ}}
$$

uniquely determining $M_{c h}$ up to a trace to be given by

$$
\mathrm{M}_{\mathrm{ch}}=-\gamma \sigma_{2}
$$

Dichroism is anisotropic absorption dependent on polarization, specified by a total attenuation parameter $\delta$ and a direction, constituting a vector $\delta$. Choosing coordinates so that $\delta=$ $\{\delta, 0\}$, this effect can be written in terms of a nonhermitian symmetric matrix $M_{d}$ as

$$
\mathbf{D}=\exp \left(\mathrm{iM}_{\mathrm{d}}\right) \mathbf{d}_{0}=\left(\begin{array}{cc}
\exp \left(\rho_{0} \delta\right) & 0 \\
0 & \exp \left(-\rho_{0} \delta\right)
\end{array}\right) \mathbf{d}_{0},
$$

which uniquely determines $M_{d}$ up to a trace to be given by

$$
\mathrm{M}_{\mathrm{d}}=-\mathrm{i} \rho_{0} \delta \sigma_{3}=-\mathrm{i} \rho_{0} \delta \cdot\left\{\sigma_{3}, \sigma_{1}\right\}
$$

where the second equality implies the extension to general coordinates. In this case the trace is a coordinate-independent absorption constant that we will neglect, though it is required to make $\operatorname{TrM}_{\mathrm{d}}<0$ so the crystal is absorbing overall. In the presence of chirality and dichroism, the two eigenpolarizations in the crystal are generally elliptical and nonorthogonal.

\section{Geometrical optics}

The theory takes its simplest form paraxially expressed in terms of simple dimensionless variables, used exclusively here, and discussed in more detail in previous papers [2-4].

Rays refracted from the exit face of the crystal have an optical path length $\Phi(\kappa, \rho, \zeta)$. This is derived paraxially by expanding to quadratic terms in $\kappa$, the small transverse part of the wavevector measured in units of $1 / w . \zeta$ is the distance along the optical axis from the nonchiral $(\gamma=0)$ focal image plane, measured in units of the diffraction length $k_{0} w^{2}$, for a monochromatic incident beam with vacuum wavenumber $k_{0}$. $\rho$ is the transverse radial coordinate measured in units of the incident beam width $w$.

Rays are defined by minimizing the optical path length according to Hamilton's principle,

$$
\nabla_{\kappa} \Phi(\kappa)=0
$$

or, equivalently, as the normals to a wave surface $\Phi+\kappa \cdot \rho$, given by

$$
\Phi_{ \pm}(\kappa, \rho, \zeta)+\kappa \cdot \rho=\frac{1}{2} \zeta \kappa^{2} \pm V(\kappa)
$$

$V(\kappa)$ is determined by the effect of the crystal's optical properties on the wave surface. In the absence of chirality or dichroism we have simply $V(\kappa)=\rho_{0} \kappa$; the wave surface has a conical point along the optic axis $\kappa=0$, responsible for internal conical refraction, and a circle of points $\kappa=$ $\rho_{0} / \zeta$ around which the normals are all parallel, responsible for an axial focal line (external conical refraction). Chirality separates the two sheets of the wave surface at the conical point and $V$ becomes

$$
V(\kappa)=\sqrt{\rho_{0}^{2} \kappa^{2}+\gamma^{2}},
$$

where, here and hereafter, we define the length of a vector by

$$
\kappa \equiv \sqrt{\kappa \cdot \kappa} .
$$

Dichroism is introduced as a symmetry-breaking perturbation [4], equivalent to a transformation to a complex wavevector:

$$
V(\kappa) \rightarrow V(\tilde{\boldsymbol{\kappa}}), \quad \tilde{\boldsymbol{\kappa}} \equiv \boldsymbol{\kappa}+\mathrm{i} \delta .
$$

The resulting wave surface has been studied previously [15] in real $\kappa$ space, where its degeneracies depend upon the ratio $\rho_{0} \delta / \gamma$. For our purposes $\boldsymbol{\kappa}$ itself can take complex values and it is more useful to make a transformation to complex ray coordinates as follows. 
A Gaussian beam can be represented as a bundle of complex rays [16], simply achieved by replacing propagation distance $\zeta$ by

$$
\zeta \rightarrow \tilde{\zeta} \equiv \zeta-\mathrm{i}
$$

It was shown in [4] that dichroism of the crystal, $\delta$, and an alignment of the beam away from the optical axis, $\kappa_{0}$ (angle from the optical axis $\left.\kappa_{0} / k w\right)$, can be described by a single parameter

$$
\mathbf{u}=\zeta \delta-\kappa_{0} .
$$

We define a complex radial ray coordinate

$$
\tilde{\rho} \equiv \rho+\mathrm{iu},
$$

(omitting here a further trivial shift of origin by the small amount $+\boldsymbol{\delta}$ ), with which the optical path length is replaced by

$$
\begin{gathered}
\Phi_{ \pm}(\boldsymbol{\kappa}, \boldsymbol{\rho}, \zeta) \rightarrow \Phi_{ \pm}(\tilde{\boldsymbol{\kappa}}, \tilde{\boldsymbol{\rho}}, \tilde{\zeta})+\Phi_{0}(\tilde{\boldsymbol{\rho}}, \tilde{\zeta}) \\
\Phi_{0}(\tilde{\boldsymbol{\rho}}, \tilde{\zeta})=\mathrm{i}\left[\boldsymbol{\delta} \cdot \tilde{\boldsymbol{\rho}}+\frac{1}{2}\left(\delta^{2}-\kappa_{0}^{2}\right)\right]+\frac{1}{2} \tilde{\zeta} \delta^{2} .
\end{gathered}
$$

The only nontrivial effect of the $\tilde{\boldsymbol{\kappa}}$-independent part $\Phi_{0}$ is an exponential amplitude slope from $\boldsymbol{\delta} \cdot \tilde{\boldsymbol{\rho}}$, understood in [4] and omitted from all of the following intensity plots. For the rest of this paper we emphasize only the $\tilde{\boldsymbol{\kappa}}$ dependence of $\Phi_{ \pm}$.

Hamilton's principle (9) can be recast as minimization with respect to $\tilde{\boldsymbol{\kappa}}$, yielding the ray equation

$$
\tilde{\boldsymbol{\rho}}_{ \pm}=\left(\tilde{\zeta} \pm \frac{\rho_{0}^{2}}{\sqrt{\rho_{0}^{2} \tilde{\kappa}^{2}+\gamma^{2}}}\right) \tilde{\boldsymbol{\kappa}}
$$

which has four sets of ray solutions, $\tilde{\boldsymbol{\kappa}}_{n}(\tilde{\boldsymbol{\rho}}, \tilde{\zeta}), n=1,2,3,4$.

The geometric ray intensity involves the Hessian determinant

$$
\left|\frac{\mathrm{d} \tilde{\rho}}{\mathrm{d} \tilde{\boldsymbol{\kappa}}}\right|=\mid \frac{\tilde{\rho}}{\tilde{\kappa}}\left(\tilde{\zeta}+\frac{(\tilde{\rho} / \tilde{\kappa}-\tilde{\zeta})^{3}}{\left(\rho_{0}^{2} / \gamma\right)^{2}}\right),
$$

summed over the four sets of rays and modulated by an exponential absorption factor, in the form

$$
I(\tilde{\boldsymbol{\rho}}, \tilde{\zeta})=\frac{1}{2} \sum_{\mathrm{n}}\left|\frac{\mathrm{d} \tilde{\boldsymbol{\rho}}}{\mathrm{d} \tilde{\boldsymbol{\kappa}}_{\mathrm{n}}}\right|^{-1} \exp \left\{\operatorname{Im}\left(\Phi\left(\tilde{\kappa}_{\mathrm{n}}\right)+\Phi_{0}\right)\right\}
$$

This is sufficient to understand the two dominant features of the intensity, namely focusing and interference. Focusing occurs where the determinant (20) vanishes, along lines in threedimensional $\{\boldsymbol{\rho}, \zeta\}$ space: (i) at the branch points

$$
\tilde{\rho}=0 \Rightarrow \rho=\rho_{\mathrm{b}} \equiv \pm \mathbf{e}_{3} \times \mathbf{u},
$$

corresponding to the complexification of the axial focal spot [4], visible near the centre of figure 1; and (ii) along a complex whisker, corresponding to the complexification of the cusped caustic surface [3]. Geometric interference occurs along anti-Stokes surfaces, where the amplitudes of complex rays are equal.

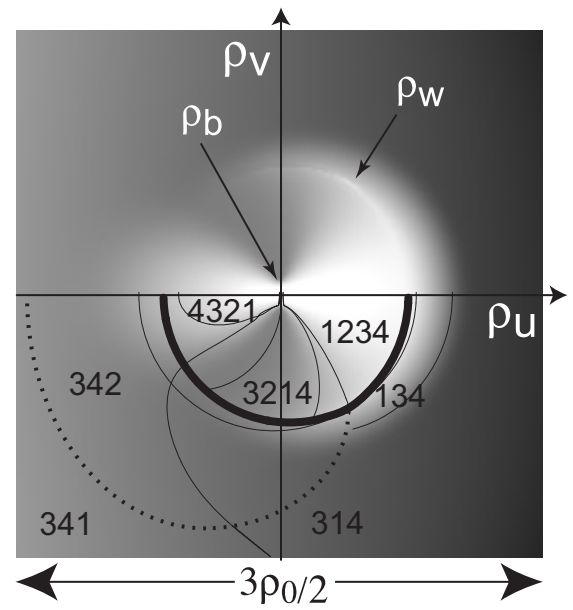

Figure 1. Logarithmic density plot of ray intensity (21) for $u=2$, $\rho_{0}=\gamma=50, \zeta=35$. The four rays are listed here in order of dominance in some key regions, with labels assigned in order of dominance in the brightest region. The dominance swaps in pairs across anti-Stokes lines (thin curves), one ray vanishes across the Stokes line (bold curve, also the bold curve in figure 4), and the ray equation (19) has a branch cut (dotted curve); these are shown only in the lower half and are symmetric about the $\rho_{\mathrm{u}}$ axis. The complex whisker $\rho_{\mathrm{w}}$ and branch points $\rho_{\mathrm{b}}$ are shown.

\subsection{The complex whisker}

The vanishing of (20) for $\tilde{\rho} \neq 0$ is satisfied by

$$
1=\left(\frac{\tilde{\zeta}}{\zeta_{\mathrm{c}}}\right)^{2 / 3}+\left(\frac{\tilde{\rho}}{\rho_{0}}\right)^{2 / 3}
$$

where

$$
\zeta_{\mathrm{c}} \equiv \rho_{0}^{2} / \gamma
$$

This defines a line of focusing between two sets of complex rays, called the complex whisker [17]. (In the case $\mathbf{u}=0$ this condition defines a horn-shaped cusped caustic surface.) As remarked in [18], the complex whisker is generally subject to exponential damping from the factors $\exp \left\{\operatorname{Im} \Phi\left(\tilde{\boldsymbol{\kappa}}_{\mathrm{n}}\right)\right\}$, reducing its significance as a visible focusing effect, though it can still be seen in figure 1 .

The whisker forms the closed loop shown in figure 2. We choose coordinates

$$
\rho=\left\{\rho_{\mathrm{u}}, \rho_{\mathrm{v}}\right\}
$$

in which $\mathbf{u}=\{u, 0\}$. By expressing $\tilde{\zeta}=\zeta-\mathrm{i}$ in complex polar form, and approximating for a small argument corresponding to $\zeta \gg 1$, the following very accurate approximation for the whisker can be found:

$$
\rho_{\mathrm{u}} \approx \frac{\rho_{0}^{2}}{u \zeta_{\mathrm{c}}}\left(\frac{\zeta_{\mathrm{c}}}{\zeta}\right)^{1 / 3}\left(1-\left(\frac{\zeta}{\zeta_{\mathrm{c}}}\right)^{2 / 3}\right)^{2}
$$

$\rho_{\mathrm{v}}^{2} \approx u^{2}-\rho_{\mathrm{u}}^{2}-\zeta_{\mathrm{c}}\left(1-\left(\frac{\zeta}{\zeta_{\mathrm{c}}}\right)^{2 / 3}\right)\left(\frac{4 \rho_{0}^{2}}{3 \zeta_{\mathrm{c}}^{3}}-\rho_{\mathrm{u}} u\left(\frac{\zeta}{\zeta_{\mathrm{c}}}\right)^{1 / 3}\right)$.

In (27) the $\rho_{0}^{2} / \zeta_{\mathrm{c}}^{3}$ term is needed for the correct shape near $\zeta=\zeta_{\mathrm{c}}$, but is small elsewhere, and the $\rho_{\mathrm{u}}^{2}$ is small near the highest arch section, while the $\rho_{\mathrm{u}}$ term is small near the lowest arch section. These considerations lead to the geometry indicated in figure 2 . 


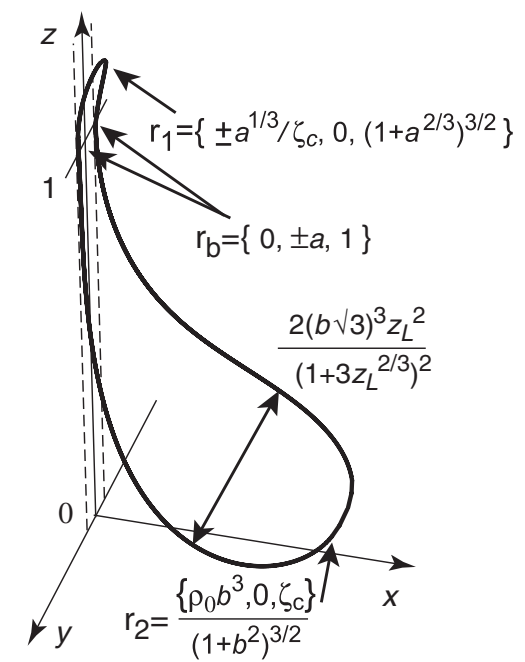

Figure 2. Geometry of the complex whisker, plotted from approximation (26) and (27) in scaled coordinates

$\{x, y, z\}=\left\{\rho_{\mathrm{u}} / \rho_{0}, \rho_{\mathrm{v}} / \rho_{0}, \zeta / \zeta_{\mathrm{c}}\right\}$, and the axial focal lines (dashed) where $\tilde{\rho}=0$. The extreme coordinates of the whisker are shown, involving $a=u / \rho_{0}, b=u \rho_{0} / \gamma=a \zeta_{\mathrm{c}}$ and $z_{L}=\left[\left(1+\sqrt{4+3 b^{2}}\right) /\left(3+3 b^{2}\right)\right]^{3 / 2}$.

\subsection{The Stokes sets}

One ray solution, $\tilde{\boldsymbol{\kappa}}_{\mathrm{m}}$, to (19), is dominant over another, $\tilde{\boldsymbol{\kappa}}_{\mathrm{n}}$, if

$$
\operatorname{Im} \Phi\left(\tilde{\boldsymbol{\kappa}}_{\mathrm{m}}\right)>\operatorname{Im} \Phi\left(\tilde{\boldsymbol{\kappa}}_{\mathrm{n}}\right) .
$$

Such dominance is exchanged across anti-Stokes surfaces defined by

$$
\operatorname{Im}\left[\Phi\left(\tilde{\boldsymbol{\kappa}}_{\mathrm{m}}\right)-\Phi\left(\tilde{\boldsymbol{\kappa}}_{\mathrm{n}}\right)\right]=0 .
$$

Figure 1 shows a typical slice through $\{\rho, \zeta\}$ space. The crowding of anti-Stokes surfaces (lines in the two-dimensional slice) mark the site of exponentially fast variation in intensity, where all of the rays are involved in pairwise exchange of dominance. Elsewhere the intensity varies slowly, being either exponentially bright or exponentially dark.

Essential to geometrical optics is the Stokes surface (nonlocal bifurcation or Maxwell set $[18,19]$ ), defined between such a pair of rays by

$$
\operatorname{Re}\left[\Phi\left(\tilde{\boldsymbol{\kappa}}_{\mathrm{m}}\right)-\Phi\left(\tilde{\boldsymbol{\kappa}}_{\mathrm{n}}\right)\right]=0 .
$$

This bounds a region in which one of the rays may not contribute and must be excluded from the sum (21). This discontinuous change generally causes no discontinuity in the intensity because the vanishing ray is subdominant (in the sense of (28)). However, the discontinuity may be seen in the geometric intensity where the Stokes and anti-Stokes surfaces of a ray pair are close together. This occurs near the complex whiskers, where the Stokes and anti-Stokes surfaces cross, and can be seen in figure 1. These discontinuities are, of course, smoothed away by diffraction in the exact wave theory.

\section{Diffraction}

We consider an incident beam represented by a superposition of plane waves, whose electric $\mathbf{D}$ vector is transverse to the optic axis and given by

$$
\mathbf{D}_{0}(\boldsymbol{\rho})=\frac{1}{2 \pi} \iint \mathrm{d} \boldsymbol{\kappa} \exp (\mathrm{i} \kappa \cdot \boldsymbol{\rho}) a(\boldsymbol{\kappa}) \mathbf{d}_{0},
$$

where $a(\boldsymbol{\kappa})$ is the Fourier transform of the incident beam profile and $\mathbf{d}_{0}$ is the incident beam's polarization. For a Gaussian beam incident along the optical axis,

$$
\begin{gathered}
a(\kappa)=\exp \left(-\frac{1}{2} \kappa^{2}\right), \\
\mathbf{D}_{0}(\rho)=\exp \left(-\frac{1}{2} \rho^{2}\right) \mathbf{d}_{0},
\end{gathered}
$$

and a misaligned beam is given by $a\left(\boldsymbol{\kappa}-\boldsymbol{\kappa}_{0}\right)$. In the absence of dichroism, the field diffracted by the crystal is given in terms of a $2 \times 2$ Hermitian matrix operator $F$ that transforms a plane wave through the crystal,

$$
\mathbf{D}(\boldsymbol{\rho}, \zeta)=\frac{1}{2 \pi} \iint \mathrm{d} \boldsymbol{\kappa} \exp \{-\mathrm{iF}(\boldsymbol{\kappa}, \boldsymbol{\rho}, \zeta)\} a(\boldsymbol{\kappa}) \mathbf{d}_{0} .
$$

The phases $\Phi_{ \pm}(\boldsymbol{\kappa}, \boldsymbol{\rho}, \zeta)$ are the eigenvalues of $\mathrm{F}$, so we can write

$$
\mathrm{F}=\Phi_{+} \mathrm{K}_{+}+\Phi_{-} \mathrm{K}_{-}, \quad \mathrm{K}_{ \pm} \equiv \frac{1}{2}\left(1 \pm \frac{\mathrm{V} \cdot \mathrm{S}}{V}\right)
$$

This fact, combined with (3) and (6), defines (up to a trace)

$$
\mathrm{F}(\boldsymbol{\kappa}, \boldsymbol{\rho}, \zeta)=-\kappa \cdot \rho+\frac{1}{2} \zeta \kappa^{2}+\mathbf{V}(\kappa) \cdot \mathrm{S},
$$

where

$$
\mathbf{V}(\boldsymbol{\kappa}) \equiv\left\{\rho_{0} \boldsymbol{\kappa}, \boldsymbol{\gamma}\right\},
$$

and

$$
\begin{aligned}
\mathrm{S} & \equiv\left\{\sigma_{3}, \sigma_{1}, \sigma_{2}\right\} \\
& =\left\{\left(\begin{array}{cc}
1 & 0 \\
0 & -1
\end{array}\right),\left(\begin{array}{ll}
0 & 1 \\
1 & 0
\end{array}\right),\left(\begin{array}{cc}
0 & -\mathrm{i} \\
\mathrm{i} & 0
\end{array}\right)\right\} .
\end{aligned}
$$

Dichroism defined by (7), and misalignment of the beam, are introduced via the transformations (13), (17), giving

$$
\begin{gathered}
\mathrm{F}(\boldsymbol{\kappa}, \boldsymbol{\rho}, \zeta) \rightarrow \mathrm{F}(\tilde{\boldsymbol{\kappa}}, \tilde{\boldsymbol{\rho}}, \tilde{\zeta})+\mathrm{F}_{0}(\tilde{\boldsymbol{\rho}}, \tilde{\zeta}) \\
\mathrm{F}_{0}(\tilde{\boldsymbol{\rho}}, \tilde{\zeta})=\mathrm{i}\left[\boldsymbol{\delta} \cdot \tilde{\boldsymbol{\rho}}+\frac{1}{2}\left(\delta^{2}-\kappa_{0}^{2}\right)\right]+\frac{1}{2} \tilde{\zeta} \delta^{2}
\end{gathered}
$$

Thus

$$
\begin{aligned}
& \mathbf{D}(\tilde{\boldsymbol{\rho}}, \tilde{\zeta})=\frac{\exp \left(-\mathrm{iF}_{0}\right)}{\tilde{\zeta}} \\
& \times {\left[C_{0}\left(\frac{\tilde{\rho}}{\sqrt{\tilde{\zeta}}} ; \frac{\rho_{0}}{\sqrt{\tilde{\zeta}}}, \gamma\right) 1+\left\{\frac{\tilde{\rho}}{\tilde{\rho}} C_{1}\left(\frac{\tilde{\rho}}{\sqrt{\tilde{\zeta}}} ; \frac{\rho_{0}}{\sqrt{\tilde{\zeta}}}, \gamma\right)\right.\right.} \\
&\left.\left.C_{2}\left(\frac{\tilde{\rho}}{\sqrt{\tilde{\zeta}}} ; \frac{\rho_{0}}{\sqrt{\tilde{\zeta}}}, \gamma\right)\right\} \cdot \mathrm{S}\right] \mathbf{d}_{0} .
\end{aligned}
$$

The $\tilde{\boldsymbol{\kappa}}$-independent part $\mathrm{F}_{0}$ constitutes the same exponential amplitude ramp as $\Phi_{0}$. We are interested in the content of the diffraction integrals,

$$
\begin{gathered}
C_{0}\left(r ; r_{0}, \gamma\right)=\int_{0}^{\infty} \mathrm{d} \tau \tau \exp \left(-\frac{1}{2} \mathrm{i} \tau^{2}\right) \mathrm{J}_{0}(r \tau) \cos \sqrt{r_{0}^{2} \tau^{2}+\gamma^{2}} \\
C_{1}\left(r ; r_{0}, \gamma\right)=\frac{r_{0}}{\gamma} \frac{\partial^{2}}{\partial r \partial \gamma} C_{0}\left(r ; r_{0}, \gamma\right) \\
C_{2}\left(r ; r_{0}, \gamma\right)=\mathrm{i} \frac{\partial}{\partial \gamma} C_{0}\left(r ; r_{0}, \gamma\right)
\end{gathered}
$$



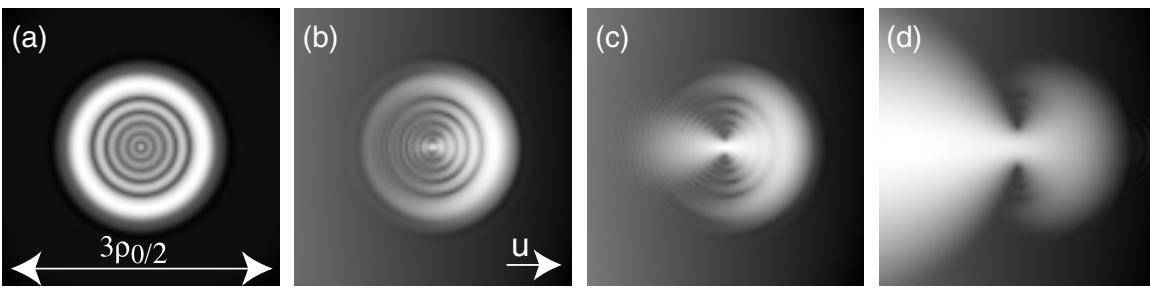

Figure 3. Logarithmic density plot of wave intensity in the $\zeta=\rho_{0} / 3$ plane with $\rho_{0}=\gamma=50$ and $u$ values: (a) 0 , (b) 1 , (c) 2 , (d) 5 . The direction of $\mathbf{u}$ is indicated in (b).
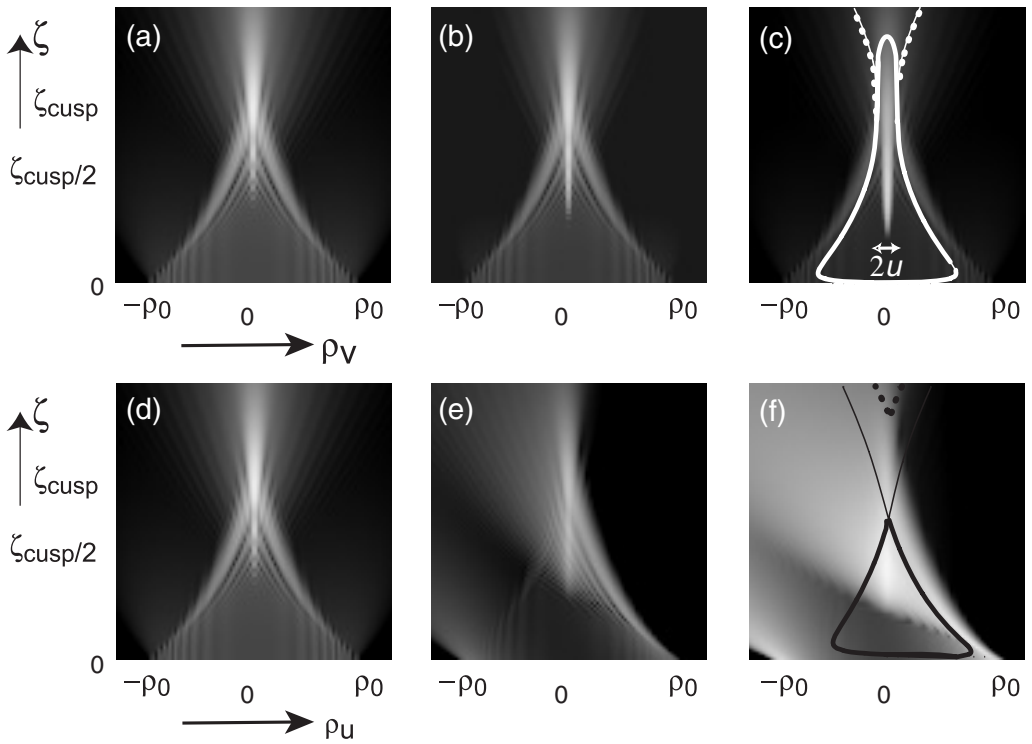

Figure 4. Logarithmic density plot of wave intensity for $\rho_{0}=\gamma=50$. (a)-(c) show the $\rho_{\mathrm{u}}=0$ plane for $u$ values: (a) 0 , (b) 1 , (c) 2 . (d)-(f) show the corresponding values in the $\rho_{\mathrm{v}}=0$ plane. (c) and (f) show the intersection of three distinct Stokes surfaces (thick, thin, and dotted curves) with these planes.

The result is identical to that in $[20,3]$ for a transparent crystal, but with the complex coordinate $\tilde{\rho}$ replacing $\rho$.

These integrals cannot be expressed in closed form, but have been integrated numerically here using Mathematica ${ }^{\mathrm{TM}}$ to produce paraxially exact simulations of the diffracted light intensity, given by

$$
I(\tilde{\boldsymbol{\rho}}, \tilde{\zeta})=\mathbf{D}(\tilde{\boldsymbol{\rho}}, \tilde{\zeta})^{*} \cdot \mathbf{D}(\tilde{\boldsymbol{\rho}}, \tilde{\zeta})
$$

We are concerned primarily with the action of the crystal rather than the effects of the incident polarization, so we consider unpolarized incident beams, for which the intensity becomes

$I_{\text {unpol }}=\frac{\exp \left(2 \operatorname{Im~} \mathrm{F}_{0}\right)}{1+\zeta^{2}}\left(\left|C_{0}\right|^{2}+\frac{\tilde{\boldsymbol{\rho}}^{*} \cdot \tilde{\boldsymbol{\rho}}}{|\tilde{\rho}|^{2}}\left|C_{1}\right|^{2}+\left|C_{2}\right|^{2}\right)$.

Figure 3 shows density plots of light intensity for varying $u$, in a plane beyond the crystal, with figure 3(c) corresponding to the ray intensity in figure 1 . The bright rings in 3(a) are associated with the caustic ring of chiral conical refraction, with a bright focal spot at the centre. As $u$ increases the focal spot spreads out between the branch lines at $\rho=\{0, \pm u\}$ and the overall intensity is consistent with the ray intensity derived in section 2 with two exceptions. The divergence at the branch points is removed, though this is actually too localized in the ray intensity to be observed except under considerable magnification. Also, the brightening associated with the complex whisker is not only softened by diffraction, but insignificant against the background of exponential variation, which is already part of the ray intensity.

The wave interference fringes, rings for $u=0$ becoming arcs as $u$ increases, are eventually swamped by the exponential gradient in the ray intensity. Figure 4 shows the intensity in the coordinate planes perpendicular to those in figures 3(a)-(c). The intensity is symmetric about $\rho_{\mathrm{v}}=0$ and, in the $\rho_{\mathrm{v}}-\zeta$ plane perpendicular to $\mathbf{u}$, the only significant effect is the spreading of the axial focal spot. The $\rho_{\mathrm{u}}-\zeta$ plane is more dramatic, showing the exponential gradient eventually swamping both the interference fringes, and the axial focal spot, as $u$ increases.

Figure 5 develops the three-dimensional intensity for the case $u=2$.

\section{Asymptotics}

\subsection{Stationary phase}

The ray optics in section 2 arises in the geometric limit of the diffraction integrals for thick crystals, that is $\rho_{0} \gg 1$. The complex rays are endowed with phase by considering them more precisely as the saddlepoints of the integrand in (42). 

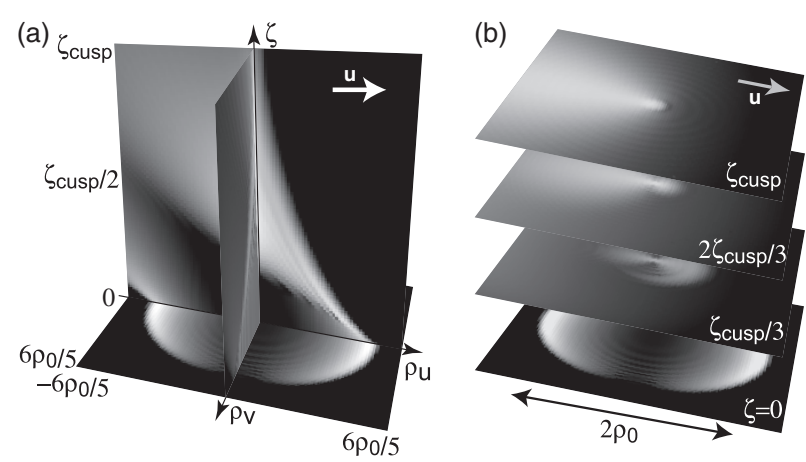

Figure 5. Wave intensity sections in three dimensions for $\rho_{0}=\gamma=50, u=2$ : (a) in the $\rho_{\mathrm{u}}=0$ plane, the $\rho_{\mathrm{v}}=0$ symmetry plane and the $\zeta=0$ focal image plane; and (b) in constant $\zeta$ planes as shown. The direction of the complexifying vector $\mathbf{u}$ is shown.

At the saddlepoints $\tilde{\boldsymbol{\kappa}}_{\mathrm{n}}$ the phases $\Phi_{ \pm}$can be expressed more simply as

$$
\Phi_{ \pm}\left(\tilde{\kappa}_{\mathrm{n}}\right)=\Phi_{\mathrm{n}} \equiv-\tilde{\kappa}_{\mathrm{n}} \tilde{\rho}+\frac{1}{2} \tilde{\zeta} \tilde{\kappa}_{\mathrm{n}}^{2}+\frac{\rho_{0}^{2}}{\tilde{\rho} / \tilde{\kappa}_{\mathrm{n}}-\tilde{\zeta}},
$$

and the second derivative as

$$
\frac{\partial^{2}}{\partial \tilde{\kappa}_{x}^{2}} \Phi_{ \pm}\left(\tilde{\kappa}_{\mathrm{n}}\right)=\Phi_{\mathrm{n}}^{\prime \prime} \equiv \tilde{\zeta}+\frac{\left(\tilde{\rho} / \tilde{\kappa}_{\mathrm{n}}-\tilde{\zeta}\right)^{3}}{\zeta_{\mathrm{c}}^{2}}
$$

when $\tilde{\rho}$ is taken to lie along the $x$ direction, so the corresponding $y$ derivative is trivially zero. Then

$$
C_{\mathrm{m}}(\rho, \zeta) \approx \frac{1}{2} \tilde{\zeta} \sum_{\mathrm{n}} c_{\mathrm{m}}\left(\tilde{\kappa}_{\mathrm{n}}\right) \sqrt{\frac{\tilde{\kappa}_{\mathrm{n}}}{\tilde{\rho} \Phi_{\mathrm{n}}^{\prime \prime}}} \mathrm{e}^{-\mathrm{i} \Phi_{\mathrm{n}}},
$$

(correcting an erroneous omission of the $\sqrt{\kappa}$ factor in [3]), where

$$
\begin{gathered}
c_{0}(\tilde{\kappa})=1, \quad c_{1}(\tilde{\kappa})=\frac{\tilde{\kappa}}{\rho_{0}}\left(\frac{\tilde{\rho}}{\tilde{\kappa}}-\tilde{\zeta}\right), \\
c_{2}(\tilde{\kappa})=\frac{\gamma}{\rho_{0}^{2}}\left(\frac{\tilde{\rho}}{\tilde{\kappa}}-\tilde{\zeta}\right) .
\end{gathered}
$$

The argument of the square root in (47) requires careful consideration. The obstruction to an explicit expression valid for all $\{\boldsymbol{\rho}, \zeta\}$ is the requirement that the argument vary smoothly throughout a given region (i.e. not jumping at $\arg \Phi=\pi$, for example), except at a single branch cut surface where the subdominant and noncontributing solutions $\tilde{\boldsymbol{\kappa}}_{n}$ must swap, as shown in figure 1 . This can, however, easily be solved, for any specified region.

With these conditions the geometric intensity is plotted in figure 6 for $u=2$. This shows how the ray intensity in figure 1 is decorated by the interference shown in 3(c). The dark region where anti-Stokes lines crowd in figure 1 marks the region of greatest interference between the four different geometric rays. Along the $\rho_{\mathrm{v}}=0$ axis, this geometric intensity is indistinguishable from the exact, and figure 7 shows how, at a typical value of $\zeta$, the bright Airy fringes associated with the caustic for $u=0$, and the axial spot, are swamped by exponential damping as $u$ increases.

Finally, figures 8(b) and (e) also show the geometric intensity near the complexified cusp.

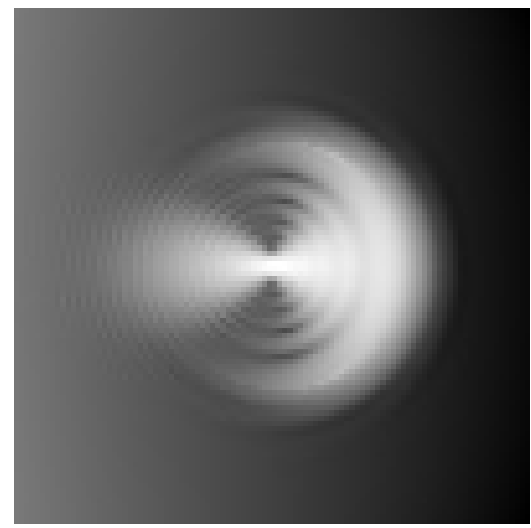

Figure 6. Logarithmic density plot of geometrical optics intensity (47) corresponding to figures 1 and 3(c), endowing rays with phase.

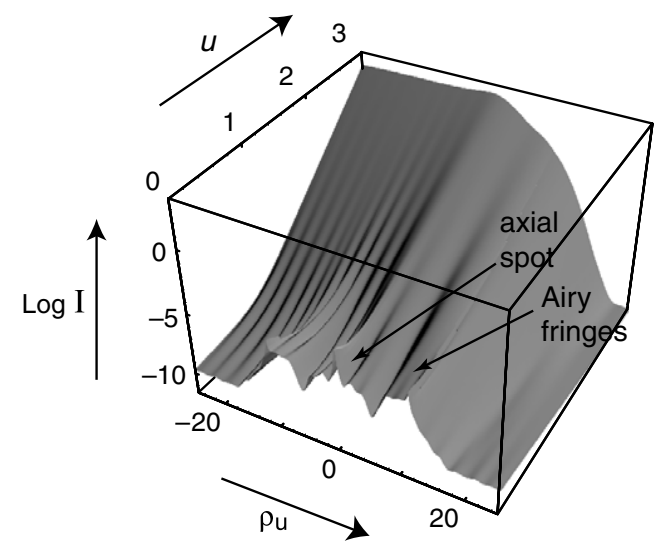

Figure 7. Logarithmic density plot of intensity on the $\rho_{\mathrm{v}}=0$ axis at $\zeta=25$ for $\rho_{0}=\gamma=50$. As $u$ increases from zero the symmetric Airy fringes associated with the caustic, and the bright axial spot, are overcome by an exponential gradient.

\subsection{The complexified spun cusp}

The cusp is identified by the intersection of two focal lines, where the third derivative of $\Phi(\tilde{\boldsymbol{\kappa}})$ vanishes. This cannot be satisfied exactly with the complex $\tilde{\zeta}$, but the complex whisker (23) and branch lines (22) do approach within the small distance $1 / \zeta_{\mathrm{c}}$, at the two points $z_{\mathrm{b}}$ in figure 2 , with coordinates

$$
\left\{0, \pm u, \zeta_{\mathrm{c}}\right\}
$$

near which the diffraction integrals (42) can be expressed approximately in terms of the 'spun cusp' integral

$$
\mathrm{J}[\mu, v]=\int_{0}^{\infty} \mathrm{d} s s \exp \left\{-\mathrm{i}\left(s^{4}+\mu s^{2}\right)\right\} \mathrm{J}_{0}(\nu s),
$$

by introducing a new integration variable

$$
\tau=s \sqrt{2\left(s^{2}+\gamma \sqrt{2 \tilde{\zeta}} / \rho_{0}\right)} \approx s \sqrt{2 \gamma \sqrt{2 \tilde{\zeta}} / \rho_{0}} .
$$



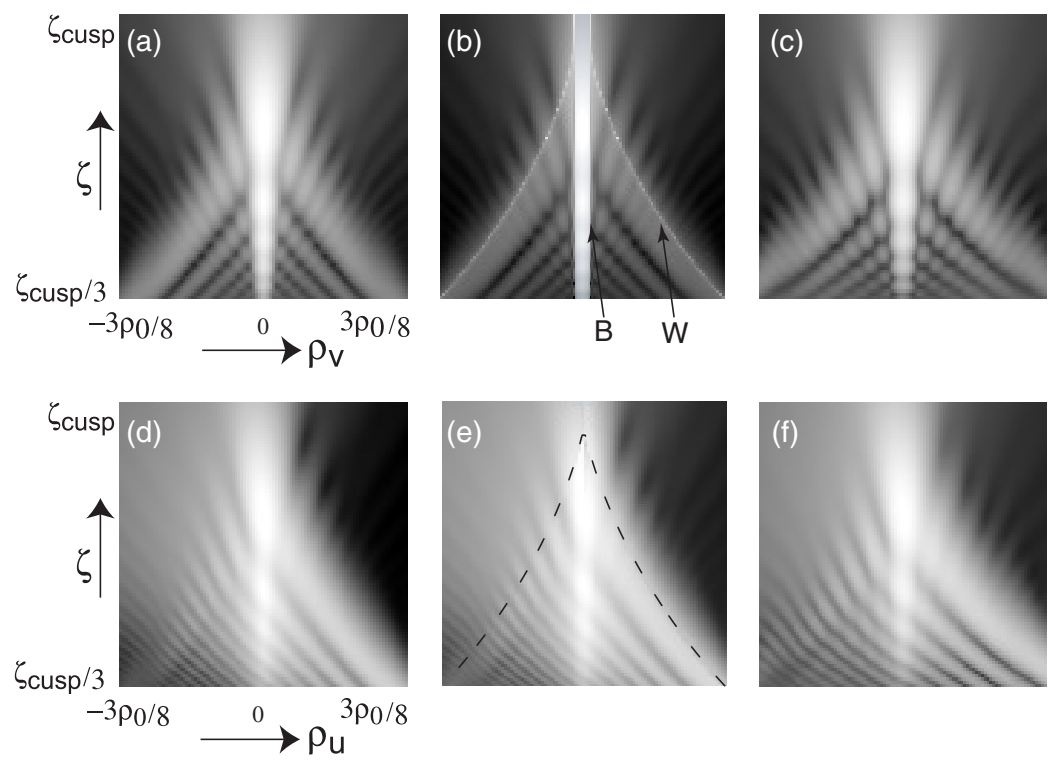

Figure 8. (a)-(c) Logarithmic density plot of intensity near the complexified spun cusp for $\rho_{0}=\gamma=50$ and $u=2$ in the $\rho_{\mathrm{u}}=0$ plane: (a) exact from (42); (b) geometrical optics (47) for $\zeta \gg 1$, which diverges along the complex whiskers $\mathrm{W}$ and $\tilde{\rho}=0$ line B; (c) spun cusp approximation (52), smoothing the discontinuities but decorating the wrong caustic far from the cusp. (d)-(f) Intensity in the $\rho_{\mathrm{v}}=0$ plane corresponding to (a)-(c), with the Stokes line (dashed) shown in (b).

The result is

$$
\begin{gathered}
C_{0}\left(r ; r_{0}, \gamma\right) \approx 2\left\{\left(\frac{v}{2 r}\right)^{2}+\mathrm{i} \frac{\partial}{\partial \mu}\right\} \sum_{ \pm} \exp ( \pm \mathrm{i} \gamma) \mathrm{J}\left(\mu_{ \pm}, v\right) \\
C_{1}\left(r ; r_{0}, \gamma\right) \approx \frac{\mathrm{i} v}{r} \sqrt{2} \frac{\partial}{\partial v} \sum_{ \pm} \pm \exp ( \pm \mathrm{i} \gamma) \mathrm{J}\left(\mu_{ \pm}, v\right) \\
C_{2}\left(r ; r_{0}, \gamma\right) \approx-\frac{v^{2}}{2 r^{2}} \sum_{ \pm} \pm \exp ( \pm \mathrm{i} \gamma) \mathrm{J}\left(\mu_{ \pm}, v\right),
\end{gathered}
$$

where

$$
\mu_{ \pm} \equiv \sqrt{2}\left(\gamma / r_{0} \mp r_{0}\right), \quad v \equiv r \sqrt{2 \gamma \sqrt{2} / r_{0}} .
$$

Figures 8(c), (f) show that this approximation correctly captures the intensity near $\zeta=\zeta_{\mathrm{c}}$, though the caustic differs from the exact one for smaller $\zeta$.

Emanating from the cusp for real $\mu$ and $v$ is the caustic

$$
v^{2}=\left(-\frac{2}{3} \mu_{+}\right)^{3} \Rightarrow\left(\frac{\tilde{\rho}}{\rho_{0}}\right)^{2}=-\frac{\zeta_{\mathrm{c}}}{\tilde{\zeta}}\left(\frac{2}{3} \frac{\tilde{\zeta}-\zeta_{\mathrm{c}}}{\zeta_{\mathrm{c}}}\right)^{3}
$$

If we consider that $\zeta \gg 1$ so $\tilde{\zeta} \approx \zeta$, for which the cusp lies at $\zeta=\zeta_{\mathrm{c}}$, the key effect of dichroism is to make the spun cusp argument $v$ complex (leaving $\mu$ real). Near this 'complexified cusp', the caustic, as already seen, is replaced by the complex whisker, given locally by

$$
\rho_{\mathrm{u}} \approx 0, \quad \rho_{\mathrm{v}} \approx \pm u \mp \frac{\rho_{0}^{2}}{2 u}\left(\frac{2}{3} \frac{\zeta-\zeta_{\mathrm{c}}}{\zeta_{\mathrm{c}}}\right)^{3} .
$$

Figures 8(b), (e) show the geometrical intensity with $\tilde{\zeta}$ replaced by $\zeta$, in which this complex whisker can be clearly seen.

\section{Concluding remarks}

The intensity of light diffracted from a dichroic chiral birefringent crystal can be understood in terms of geometrical optics. The complex rays necessary in the presence of absorption pose no intuitive obstacle, even though they do not represent any ray path in real space, defining paths conserving the complex wavevector $\tilde{\kappa}=\kappa+\mathrm{i} \delta$. They yield a detailed and simple understanding of the dominant features in the geometric intensity, including focusing and the exponential gradients expected from absorption. Wave interference effects can be added to the geometrical optics intensity similarly to dealing with real rays, providing a complete understanding of the diffracted light intensity. The divergence along the complex whisker and branch point focal lines, which are physically smoothed away by diffraction, could be dealt with analytically by a uniform mapping to a function with the same critical structure, as in the appendix of [3], expressing the diffraction integrals in terms of the Airy function of a complex argument.

The geometric intensity in the presence of chirality can be evaluated numerically with ease, but an explicit analytic expression is complicated by the need to solve the quartic ray equation. Nevertheless, this poses little obstruction to a detailed geometric understanding. In the presence of dichroism, the obvious focal lines may not be the dominant features of even the geometrical intensity. Instead, the intensity is dominated by the exchange of dominance of rays across antiStokes surfaces, the sites of geometric interference between complex rays.

In the absence of chirality the anti-Stokes set forms a dark cylindrical surface [3]. The exact circularity of the surface results from the equivalence implied by (14), between a Gaussian beam, and a bundle of rays whose propagation distance is shifted by an imaginary constant. In the method of stationary phase this amounts to incorporating the beam 
profile into the phase, because it cannot be treated as slowly varying. For more slowly varying beams (e.g. light refracted from a pinhole) the analysis from [4] can be expected to hold approximately with the imaginary shift in propagation distance removed. This changes the dark cylinder into a pair of dark brushes sprouting symmetrically outwards from the lines $\tilde{\rho}=$ 0 , corresponding to the cylinder radius in [3] becoming infinite. With chirality included, neglecting the imaginary shift makes all terms in (23) real, forcing the complex whisker to lie in the plane of the lines $\tilde{\rho}=0$ (the $x=\rho_{\mathrm{u}}=0$ plane in figure 2 ), as is seen in the approximate cusp intensity in figures 8(b), (e).

The geometric meaning of the wave surface, which has historically played an important intuitive role in conical diffraction, is unclear for complex rays. The wave surface's local singular structure (a pair of singular axes if $\delta \geqslant \gamma / \rho_{0}$, a ring of inflection points if $\left.\delta<\gamma / \rho_{0}\right)$ is not obviously apparent in the ray intensity. Therefore the haunting theorem [15], in which points of circular polarization 'haunt' the locations of the departed singular axes in $\kappa$ space as chirality is added, has no counterpart in the diffracted light field. Furthermore, the wave surface seems to offer no intuitive understanding of the duality between beam direction and dichroism for a Gaussian beam.

It is beyond the scope of this paper to discuss the various optical properties responsible for the effects contained herein. In particular, optical activity can arise through the Faraday effect and Raman optical activity, as well as from crystalline chirality and underlying molecular chirality. We maintain generality by specifying the effect only in terms of the total optical rotary power of the crystal. Though we have not considered circular dichroism it does not fundamentally alter the nature of the phenomenon and can be incorporated simply from our introductory arguments: the effect is to make $\gamma$ imaginary (leaving $\tilde{\rho}=\rho$ real), merely changing the orientation of the singular axes. The relation of $\delta$ and $\gamma$ to the dielectric tensor has previously been given in [4] and [20].

Much of conical diffraction remains unexplored experimentally. Observations near the singular axis of a biaxial dichroic crystal were made in [21] but did not concern conical diffraction. Conical diffraction experiments have been performed on transparent crystals, most extensively the nonchiral case $[22,23,2]$, which is now well understood. The chiral case was studied in [24], although the caustic structure and $\zeta$ dependence predicted in [3] remains to be investigated, and should be easy to observe. The size of the effects individually have been considered in [3] and [4], although hampered by a lack of data regarding absorption coefficients. The effects are prominent when the singular axes have angular separation on the order of $2 \delta / k_{0} w$, for example $1^{\circ}$ for a He-Ne laser with a $10 \mu \mathrm{m}$ waist width.

There are no published experimental observations, to our knowledge, which unveil the rich distance-dependent intensity structure beyond a biaxial crystal in the presence of chirality and/or dichroism, or equivalently, of the transition from conical diffraction to double refraction for transparent crystals with or without chirality. Transition in transparent crystals offers a simpler route, since dichroism imposes an extra exponential ramp across the intensity. The phenomena predicted here and in [4] pose an experimental challenge since the interference effects are faint, revealing themselves only in logarithmic intensity plots.

\section{Acknowledgments}

I thank M V Berry for many helpful discussions. My research is supported by EPSRC.

\section{References}

[1] Berry M V 2004 Conical diffraction asymptotics: fine structure of Poggendorff rings and axial spike J. Opt. A: Pure Appl. Opt. 6 289-300

[2] Berry M V, Jeffrey M R and Lunney J G 2006 Conical diffraction: observations and theory Proc. R. Soc. A 462 1629-42

[3] Berry M V and Jeffrey M R 2006 Chiral conical diffraction J. Opt. A: Pure Appl. Opt. 8 363-72

[4] Berry M V and Jeffrey M R 2006 Conical diffraction complexified: dichroism and the transition to double refraction J. Opt. A: Pure Appl. Opt. 8 1043-51

[5] Berry M V and Jeffrey M R 2007 Conical diffraction: Hamilton's diabolical point at the heart of crystal optics Prog. Opt. at press

[6] Hamilton W R 1837 Third supplement to an essay on the theory of systems of rays Trans. R. Irish Acad. 17 1-144

[7] Belskii A M and Khapalyuk A P 1978 Internal conical refraction of bounded light beams in biaxial crystals $O p t$. Spectrosc. (USSR) 44 436-9

[8] Landau L D, Lifshitz E M and Pitaevskii L P 1984 Electrodynamics of Continuous Media 2nd edn (Oxford: Pergamon)

[9] Voigt W 1905 Ueber die Wellen flaeche zweiachsiger aktiver kristalle und ueber ihre konische refraktion Phys. Z. 6 787-90

[10] Voigt W 1905 Theoretisches und Experimentalles zur Aufklaerung des optischen Verhaltens aktiver Kristalle Ann. Phys., Lpz. 18 645-94

[11] Pancharatnam S 1955 The propagation of light in absorbing biaxial crystals-i. Theoretical Proc. Indian Acad. Sci. 42 109-86

[12] Ramachandran G N and Ramaseshan S 1961 Crystal optics Handbuch der Physik vol XXV/I ed H Flügge (Berlin: Springer)

[13] Paris R B 1991 The asymptotic behaviour of Pearcey's integral for complex variables Proc. R. Soc. A 432 391-426

[14] Lloyd H 1837 On the phenomena presented by light in its passage along the axes of biaxial crystals Trans. R. Irish Acad. 17 145-58

[15] Berry M V and Dennis M R 2003 The optical singularities of birefringent dichroic chiral crystals Proc. R. Soc. A 459 1261-92

[16] Deschamps G A 1971 Gaussian beam as a bundle of complex rays Electron. Lett. 7 684-5

[17] Poston T and Stewart I N 1976 Taylor Expansions and Catastrophes (London: Pitman)

[18] Berry M V and Howls C J 1990 Stokes surfaces of diffraction catastrophes with codimension three Nonhermiticity $\mathbf{3}$ 281-91

[19] Wright F J 1980 The Stokes set of the cusp diffraction catastrophe J. Phys. A: Math. Gen. 13 2913-28

[20] Belsky A M and Stepanov M A 2002 Internal conical refraction of light beams in biaxial gyrotropic crystals Opt. Commun. $2041-6$

[21] Pancharatnam S 1955 The propagation of light in absorbing biaxial crystals_-ii. Experimental Proc. Indian Acad. Sci. 42 235-48

[22] Rajagopalan V S, Raman C V and Nedungadi T M K 1941 Conical refraction in naphthalene crystals Proc. Indian Acad. Sci. A 14 221-7

[23] Schell A J and Bloembergen N 1978 Laser studies of internal conical diffraction i. Quantitative comparison of experimental and theoretical conical intensity distribution in aragonite J. Opt. Soc. Am. 68 1093-8

[24] Schell A J and Bloembergen N 1978 Laser studies of internal conical diffraction. ii. Intensity patterns in an optically active crystal, $\alpha$-iodic acid J. Opt. Soc. Am. 68 1098-106 